\title{
Enhanced limonene production by optimizing the expression of limonene biosynthesis and MEP pathway genes in E. coli
}

Fu-Liang Du, Hui-Lei Yu, Jian-He Xu and Chun-Xiu Li

\begin{abstract}
Background: Limonene is an important monoterpene used as a chemical commodity and precursor for producing biofuels, flavor and medicinal compounds.

Results: In this paper, we engineered Escherichia coli by embedding two exogenous genes encoding a limonene synthase (LS) and a geranyl diphosphate synthase (GPPS) for production of limonene. Out of 12 E. coli strains transformed with various plasmids, the best one with p15T7-Is-gpps produced limonene with a titer of $4.87 \mathrm{mg} / \mathrm{L}$. In order to enhance the limonene production, two rate-limiting enzymes in the endogenous MEP pathway of $E$. coli, 1-deoxy-xylulose-5-phosphate synthase (DXS) and isopentenyl diphosphate isomerase (IDI), were overexpressed consecutively on vector pET21at, resulting in a production of $17.4 \mathrm{mglimonene} / \mathrm{L}$ at $48 \mathrm{~h}$.

Conclusions: After the preliminary optimization of the medium in a two-phase culture system composed of $n$-hexadecane $\left(1 / 50, V_{\text {org }} / V_{\text {aq }}\right)$, the final production of limonene was raised up to $35.8 \mathrm{mg} / \mathrm{L}$, representing approximately a 7-fold improvement compared to the initial titer.
\end{abstract}

Keywords: Limonene; Biosynthesis; Escherichia coli; MEP pathway; Geranyl pyrophosphate synthase; Limonene synthase; Two-phase culture system

\section{Background}

Terpenoid, which has more than forty thousand kinds of chemicals, is the largest family of natural products [1]. Limonene, one of the simplest monocyclic $p$-menthane (1-methyl-4-isopropylcyclohexane) type monoterpenes, has been used as a flavor or fragrance with aroma value $[2,3]$. Limonene is also an important plant monoterpene precursor of several fine chemicals, flavorings, fragrances, and pharmaceuticals such as carveol, carvone, perillyl alcohol and menthol [2-6]. In addition, the hydrogenated form of limonene can be used as fuel [7]. However, the major supply of limonene is limited to plant sources currently. Although chemical processes for monoterpene synthesis have been well documented, very few pathwayengineered processes [3,4] have been reported so far for the production of limonene. Therefore, it is promising and

\footnotetext{
* Correspondence: chunxiuli@ecust.edu.cn

Laboratory of Biocatalysis and Synthetic Biotechnology, State Key Laboratory of Bioreactor Engineering, East China University of Science and Technology, Shanghai 200237, P. R. China
}

attractive to develop a route of microbial synthesis for limonene production.

Isopentenyl diphosphate (IPP) and its isomer dimethylallyl diphosphate (DMAPP) are the two essential building blocks to synthesize all isoprenoids (terpenoids). There are two biosynthetic pathways to produce IPP and DMAPP. The first one is mevalonate pathway (MVA pathway) present in all higher eukaryotes such as Saccharomyces cerevisiae [8-10] The second one is MEP pathway starting with the reaction between pyruvate and glyceraldehyde-3phosphate to synthesize IPP and DMAPP $[9,11]$. Plants produce their terpenoids using MEP pathway, which takes place in their plastids. In addition, MEP pathway occurs in most bacteria including Escherichia coli.

Based on the IPP and DMAPP produced from the MEP pathway, limonene can be synthesized by the catalysis of two key enzymes $[4,12]$, geranyl diphosphate synthase (GPPS) and limonene synthase (LS), which were reported to occur in Abies grandis [13] and Mentha spicata [2], respectively. GPPS catalyzes the condensation between IPP and DMAPP, forming a linear diphosphate intermediate, 
geranyl diphosphate (GPP), the precursor of all the monoterpenes. On the other hand, LS catalyzes the intramolecular cyclization of GPP to give limonene (Figure 1).

Although overexpression of rate-limiting enzymes in the MEP pathway has been used to produce many terpenoids including isoprene, taxol precursor and levopimaradiene [14-19], this method has not been used in the most common engineered strain Escherichia coli to produce limonene. In a previous study, an engineered Escherichia coli, harboring a heterologous GPPS gene from Abies grandis and a LS gene from Mentha spicata, was reported to produce limonene in a titer of merely $5 \mathrm{mg} / \mathrm{L}(24 \mathrm{~h})$ [4]. A possible reason for the low limonene production might be the insufficient supply of IPP and DMAPP. In another report, an engineered Escherichia coli in which a heterologous MVA pathway was installed, can produce limonene with titers around $335 \mathrm{mg} / \mathrm{L}$ at $48 \mathrm{~h} \mathrm{[3].}$

In this paper, we engineered $E$. coli to produce (-)-limonene by expressing a GPPS, a LS, a 1-deoxy-dxylulose-5-phosphate synthase (DXS) and an isopentenyl diphosphate isomerase (IDI). A series of modifications to optimize the expression of those enzymes and the medium have been done. The final production of limonene was raised up to $35.8 \mathrm{mg} / \mathrm{L}$, representing approximately a 7 -fold improvement compared to the initial titer.

\section{Methods}

\section{Bacterial strains, genes and vectors}

Escherichia coli BL21 (DE3) (Novagen, Germany) was used for gene cloning and expression. Escherichia coli K12 MG1655 was used for amplification of $d x s$, idi, and ispA genes. The gene sequences of GPPS and LS were obtained from Abies grandis and Mentha spicata (Genbank accession numbers: AF513112 and L13459). These genes were condon optimized for E. coli and commercially synthesized in Generay (Shanghai, China). $N$-Terminal amino acids at positions 87 and 56 of GPPS and LS (plastid transit peptide) were removed [13,20]. Vectors pET-21a, pET-28a, pACYCDuet-1, pQE30 and pTrcHis2B (Novagen, Germany) were used for gene expression.

\section{Genes cloning and plasmids construction}

The PCR fragments of gpps and $l s$ were cloned into the BamHI-EcoRI and EcoRI-SalI sites of the pTrcHis2B vector to create p40Trc-ls-gpps and p40Trc-gpps-ls plasmids. The generated $l s$-gpps and gpps-ls operons from p40Trc-ls-gpps and p40Trc-gpps-ls were then cloned into pACYCDueT-1 vector through BamH I and Sal I sites to generate p15T7-ls-gpps and p15T7-gpps-ls plasmids. For constructing p40T7-ls-gpps and p40T7-gpps-ls plasmids, initially the operons $l s$-gpps and gpps-ls were inserted into pET-28a vector at BamH I and Sal I sites. The ispA ${ }^{\mathrm{S} 80 \mathrm{~F}}$, which is designated as EcGPPS here, is another geranyl pyrophosphate synthase. The method for constructing plasmids p40Trc-ls-Ecgpps, p40Trc-Ecgpps-ls, p15T7-lsEcgpps, p40T7-ls-Ecgpps, p40T7-Ecgpps-ls and p15T7Ecgpps-ls was the same as the plasmids listed above. The $d x s$-idi operon was initially constructed by cloning each of the genes from the genome of E. coli K12 MG1655. The $d x s$-idi operon was cloned into pTrcHis2B vector by the BamH I-Kpn I sites to create p40Trc-dxs-idi plasmid. The $d x s$-idi operon was sub-cloned into the pQE30 and pET-21a vectors through BamH I and Sal I sites to construct p40T5-dxs-idi and p40T7-dxs-idi plasmids, respectively. The primers used were listed in Table 1. All plasmids in this study were shown in Table 2, and all engineered $E$. coli strains and the corresponding plasmids were shown in Table 3.

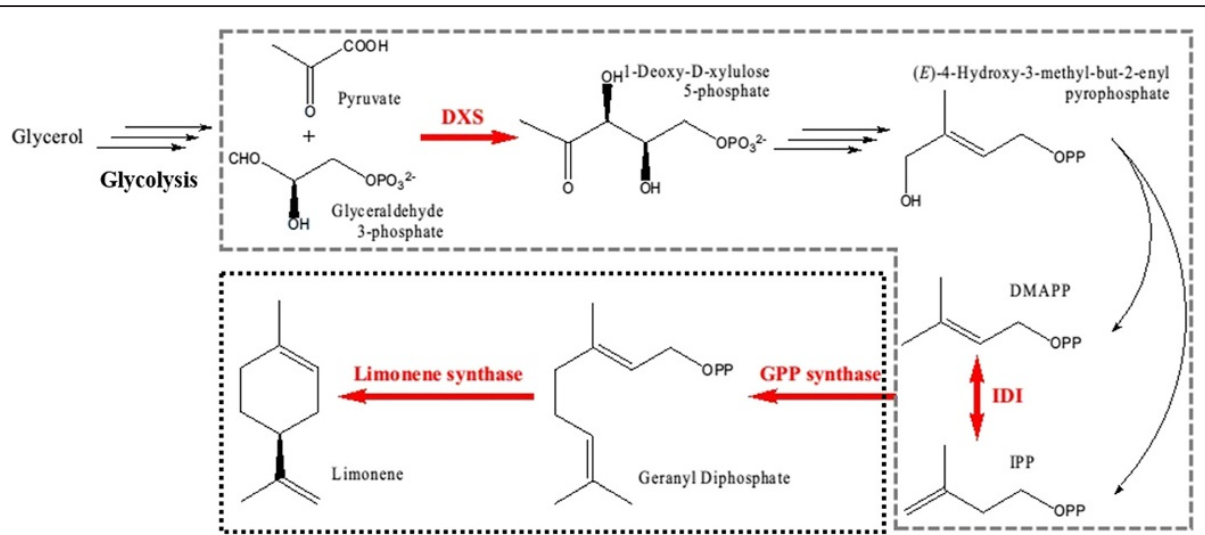

(-)-Limonene Biosynthesis Pathway

MEP Pathway

Figure 1 Engineered pathway for (-)-limonene biosynthesis in E. coli. (-)-Limonene was biosynthesized via glycolysis, the endogenous MEP pathway in E. coli and the exogenous limonene biosynthesis pathway. DXS: 1-Deoxy-d-xylulose-5-phosphate synthase; IDI: Isopentenyl diphosphate isomerase. 
Table 1 The plasmids constructed in this study

\begin{tabular}{|c|c|c|c|c|c|}
\hline Plasmids & Parent plasmids & Copies & Promoter & Antibiotic marker & Expression strength \\
\hline p40Trc-Is-gpps & pTrcHis2B & 40 & $\operatorname{TrC}$ & ampicillin & 40 \\
\hline p15T7-/s-gpps & pACYCDuet-1 & 15 & $\mathrm{~T} 7$ & chloramphenicol & 75 \\
\hline p40T7-/s-gpps & pET28a+ & 40 & $\mathrm{~T} 7$ & kanamycin & 200 \\
\hline p40Trc-gpps-1s & pTrcHis2B & 40 & $\operatorname{TrC}$ & ampicillin & 40 \\
\hline p15T7-gpps-Is & pACYCDuet-1 & 15 & $\mathrm{~T} 7$ & chloramphenicol & 75 \\
\hline p40T7-gpps-1s & pET28a+ & 40 & $\mathrm{~T} 7$ & kanamycin & 200 \\
\hline p40Trc-Is-Ecgpps & pTrcHis2B & 40 & $\operatorname{TrC}$ & ampicillin & 40 \\
\hline p15T7-/s-Ecgpps & pACYCDuet-1 & 15 & $\mathrm{~T} 7$ & chloramphenicol & 75 \\
\hline p40T7-/s-Ecgpps & pET28a+ & 40 & $\mathrm{~T} 7$ & kanamycin & 200 \\
\hline p40Trc-Ecgpps-/s & pTrcHis2B & 40 & $\operatorname{TrC}$ & ampicillin & 40 \\
\hline p15T7-Ecgpps-Is & pACYCDuet-1 & 15 & $\mathrm{T7}$ & chloramphenicol & 75 \\
\hline p40T7-Ecgpps-Is & pET28a+ & 40 & $\mathrm{T7}$ & kanamycin & 200 \\
\hline p40T7-dxs-idi & pET21a+ & 40 & $\mathrm{~T} 7$ & ampicillin & 200 \\
\hline p40T5-dxs-idi & pQE30 & 40 & T5 & ampicillin & 80 \\
\hline p40Trc-dxs-idi & pTrcHis2B & 40 & $\operatorname{Trc}$ & ampicillin & 40 \\
\hline
\end{tabular}

The expression strengths of vectors were estimated using published values of promoter strength and copy number. Promoter strengths were calculated as Trc $=1, \mathrm{~T} 5=2$, T7 = 5 (Brosius et al. [21], Brunner et al. [22]). Gene copy number was assigned by published copy numbers for origin of replication for the different plasmids used.

\section{Culture media and conditions}

Culture for limonene production was carried out in $2 \mathrm{YT}$ medium using a shaking incubator at $20^{\circ} \mathrm{C}$ and $180 \mathrm{rpm}$. Glycerol as the main carbon source, was added at $2 \%$ (w/v) concentration to all the media used in this study [23]. LB medium and TB medium were compared with

Table 2 List of engineered $E$. coli strains and the corresponding plasmids

\begin{tabular}{|c|c|c|c|c|}
\hline \multirow[t]{2}{*}{ Strain } & \multicolumn{2}{|c|}{ MEP pathway $d x s$-idi operon } & \multicolumn{2}{|c|}{$\begin{array}{c}\text { Limonene pathway Is-gpps, } \\
\text { gpps-Is, Is-Ecgpps, or } \\
\text { Ecgpps-Isoperons }\end{array}$} \\
\hline & Plasmid & $\begin{array}{l}\text { Expression } \\
\text { strength }\end{array}$ & Plasmid & $\begin{array}{c}\text { Expression } \\
\text { strength }\end{array}$ \\
\hline 1 & - & 1 & p40Trc-ls-gpps & 40 \\
\hline 2 & - & 1 & p15T7-/s-gpps & 75 \\
\hline 3 & - & 1 & p40T7-Is-gpps & 200 \\
\hline 4 & - & 1 & p40Trc-gpps-ls & 40 \\
\hline 5 & - & 1 & p15T7-gpps-ls & 75 \\
\hline 6 & - & 1 & p40T7-gpps-1s & 200 \\
\hline 7 & - & 1 & p40Trc-Is-Ecgpps & 40 \\
\hline 8 & - & 1 & p15T7-/s-Ecgpps & 75 \\
\hline 9 & - & 1 & p40T7-/s-Ecgpps & 200 \\
\hline 10 & - & 1 & p40Trc-Ecgpps-ls & 40 \\
\hline 11 & - & 1 & p15T7-Ecgpps-ls & 75 \\
\hline 12 & - & 1 & p40T7-Ecgpps-Is & 200 \\
\hline 13 & p40T7-dxs-idi & 200 & p15T7-/s-gpps & 75 \\
\hline 14 & p40T5-dxs-idi & 80 & p15T7-Is-gpps & 75 \\
\hline 15 & p40Trc-dxs-idi & 40 & p15T7-/s-gpps & 75 \\
\hline
\end{tabular}

For a full plasmids description, see Table 1.
2YT medium as basis media for limonene production. The addition of pyruvate as an auxiliary carbon source has been reported to increase terpenoid production [24]. Ampicillin $(100 \mu \mathrm{g} / \mathrm{mL})$, kanamycin $(50 \mu \mathrm{g} / \mathrm{mL})$ and chloramphenicol $(50 \mu \mathrm{g} / \mathrm{mL})$ were added to the culture as required. Cell culture was carried out in $100 \mathrm{~mL}$ of medium, and growth was determined by measuring the optical density at $600 \mathrm{~nm}\left(\mathrm{OD}_{600}\right)$. For the two-phase culture for limonene production, IPTG was added when $\mathrm{OD}_{600}$ reached about 0.7 , and $2 \mathrm{~mL}$ of $n$-hexadecane was layered over $100 \mathrm{~mL}$ of culture medium. The assay for glycerol was conducted by the Nash reagent [25].

\section{Analysis of limonene}

In the two-phase culture system with $n$-hexadecane overlay, limonene was extracted with the upper $n$-hexadecane phase. Then $n$-hexadecane containing the limonene was collected and centrifuged at $14,000 \mathrm{rpm}$ for $10 \mathrm{~min}$. The $n$-hexadecane extracts were analyzed with GC-MS (QP2010, Shimadzu, Kyoto, Japan) equipped with an InertCap DB-5 ms column. Limonene from splitless $1 \mu \mathrm{L}$ injection was separated using a GC oven temperature program of $50^{\circ} \mathrm{C}$ for $3 \mathrm{~min}$, followed by a $10^{\circ} \mathrm{C} / \mathrm{min}$ ramp to $250^{\circ} \mathrm{C}$. Injector and MS quadrupole detector temperatures were $250^{\circ} \mathrm{C}$ and $150^{\circ} \mathrm{C}$, respectively. To increase the sensitivity and selectivity of detection, the MS was operated in selected ion-monitoring (SIM) mode using ions of 136, 68, and $93 \mathrm{~m} / \mathrm{z}$, which represent the molecular ion and two abundant fragmental ions of limonene. 
Table 3 Primers used in this work

\begin{tabular}{|c|c|}
\hline Primers & Sequences of oligonucleotides $\left(5^{\prime}-3^{\prime}\right)$ \\
\hline $\mathrm{dxs}\left(\mathrm{s}^{1}\right)$ & GGGAATTCCATATGAGTTTTGATATTGCCAA \\
\hline$d x s\left(a^{2}\right)$ & GCGAATTCTTATGCCAGCCAGGCCTTGATTITG \\
\hline idi (s) & CGGAATTCGAAGGAGATATACATATGCAAACGGAACACGTCATTTATTG \\
\hline idi (a) & GCGCTCGAGGCTCACAACCCCGGCAAATGTCGG \\
\hline dxsidiBamHI(s) & CGCGGATCCGATGAGTITTGATATTGCCAA \\
\hline dxsidiKpnl (a) & CGGGGTACCGCTCACAACCCCGGCAAATGTCGG \\
\hline dxsidiBamH I 1(s) & CGCGGATCCATGAGTITTGATATTGCCAA \\
\hline LSBamH I(s) & CGCGGATCCGATGGAACGTCGTAGCGGTAA \\
\hline LS(a) & CGTAGAATTCTTATGCAAATGGTTCAAACA \\
\hline GPPS(S) & CCGGAATTCGAAGGAGATATACATATGTTCGACTTCAACAAATAC \\
\hline GPPS(A) & ACGCGTCGACTCAGTTCTGACGGAATGCAAC \\
\hline GPPSBamH I(s) & CGCGGATCCGATGTTCGACTTCAACAAATAC \\
\hline GPPSBamH I 1(s) & CGCGGATCCATGTTCGACTTCAACAAATAC \\
\hline GPPS1(A) & CCGGAATTCTCAGTTCTGACGGAATGCAAC \\
\hline $\mathrm{LS1}(\mathrm{S})$ & CCGGAATTCGAAGGAGATATACATATGGAACGTCGTAGCGGTAA \\
\hline LS1 (a) & CCGGAATTCTTATGCAAATGGTTCAAACA \\
\hline S81F-rev & AATCATCATGAATTAAAAAGTAAGCGTGGATACAC \\
\hline S81F-for & GTGTATCCACGCTTACTITTAATTCATGATGATT \\
\hline EcFPPSBamHls & CGCGGATCCGATGGACTTTCCGCAGCAACT \\
\hline EcFPPSEcoRla & CCGGAATTCTTATTTATTACGCTGGATG \\
\hline LSEcoRISDs & CGTAGAATTCAGAAGGAGATATACATATGGAACGTCGTAGCGGTAA \\
\hline EcFPPSEcoRIs & CGTAGAATTCAGAAGGAGATATACATATGGACTTTCCGCAGCAACT \\
\hline EcFPPSall & ACGCGTCGACTTATTTATTACGCTGGATG \\
\hline
\end{tabular}

${ }^{1}:$ Forward primer;

${ }^{2}$ :Reverse primer.

\section{Results}

\section{Optimizing the expression of limonene biosynthesis} genes in E. coli

(-)-Limonene can be produced by introducing the gpps and $l s$ genes into E. coli. Previously, IspA ${ }^{\mathrm{S} 80 \mathrm{~F}}$ has been known to make the ispA variant synthesize GPP only [26]. We cloned the ispA genes from E. coli, and got the IspA ${ }^{\mathrm{S} 80 \mathrm{~F}}$ gene Ecgpps. Polycistronic operons consisting of gpps and ls or Ecgpps and $l s$, in which the genes can be assembled as one transcriptional unit, were constructed based on pTrcHis2B, pACYCDuet-1 and pET28a+. Then totally twelve limonene synthesis plasmids were constructed (Table 2) and introduced into the $E$. coli, respectively. Those twelve recombinant $E$. coli cell lines, each harboring one limonene biosynthesis plasmid, were cultured in 2YT medium for $48 \mathrm{~h}$. The cells were cultured with $250 \mu \mathrm{M}$ IPTG induction. As shown in Figure 2, only trace amounts of limonene were detected in the culture of E. coli harboring Ecgpps-ls operon or gpps-ls operon. While the four recombinant strains harboring ls-Ecgpps operon and ls-gpps operon (strain \#2, \#7, \#8 and \#9) could produce a quite significant amount of limonene, indicating that the limonene synthase gene should be put before the gene of geranyl diphosphate synthase when they were coexpressed in one plasmid. Moreover, the strain \#2 harboring the ls-gpps operon could produce more limonene than the other strains (strain \#7, \#8 and \#9) harboring the ls-Ecgpps operon. This result showed that GPPS was more suitable for monoterpene biosynthesis compared to EcGPPS. In addition, for the better expression of $l s$-gpps operon, a moderate expression strength (Table 2) was good for E. coli to produce limonene. We also found that the cell mass was greatly associated with the limonene production (Figure 2, C). This phenomenon indicated that the expression of plasmid p15T7-ls-gpps, which could affect the main metabolism, was conducive to cell growth. As a result, the limonene synthesis plasmid p15T7-ls-gpps was therefore selected for limonene production in further experiments. This optimized limonene pathway plasmid could lead the recombinant $E$. coli to accumulate $4.87 \mathrm{mg} / \mathrm{L}$ of limonene or $0.863 \mathrm{mg} / \mathrm{g}_{\mathrm{DCW}}$ after $48 \mathrm{~h}$ of cultivation.

\section{Overexpression of rate-limiting enzymes in the endogenous MEP pathway}

The limonene building blocks, IPP and DMAPP, can be synthesized in E. coli via the endogenous MEP pathway. 


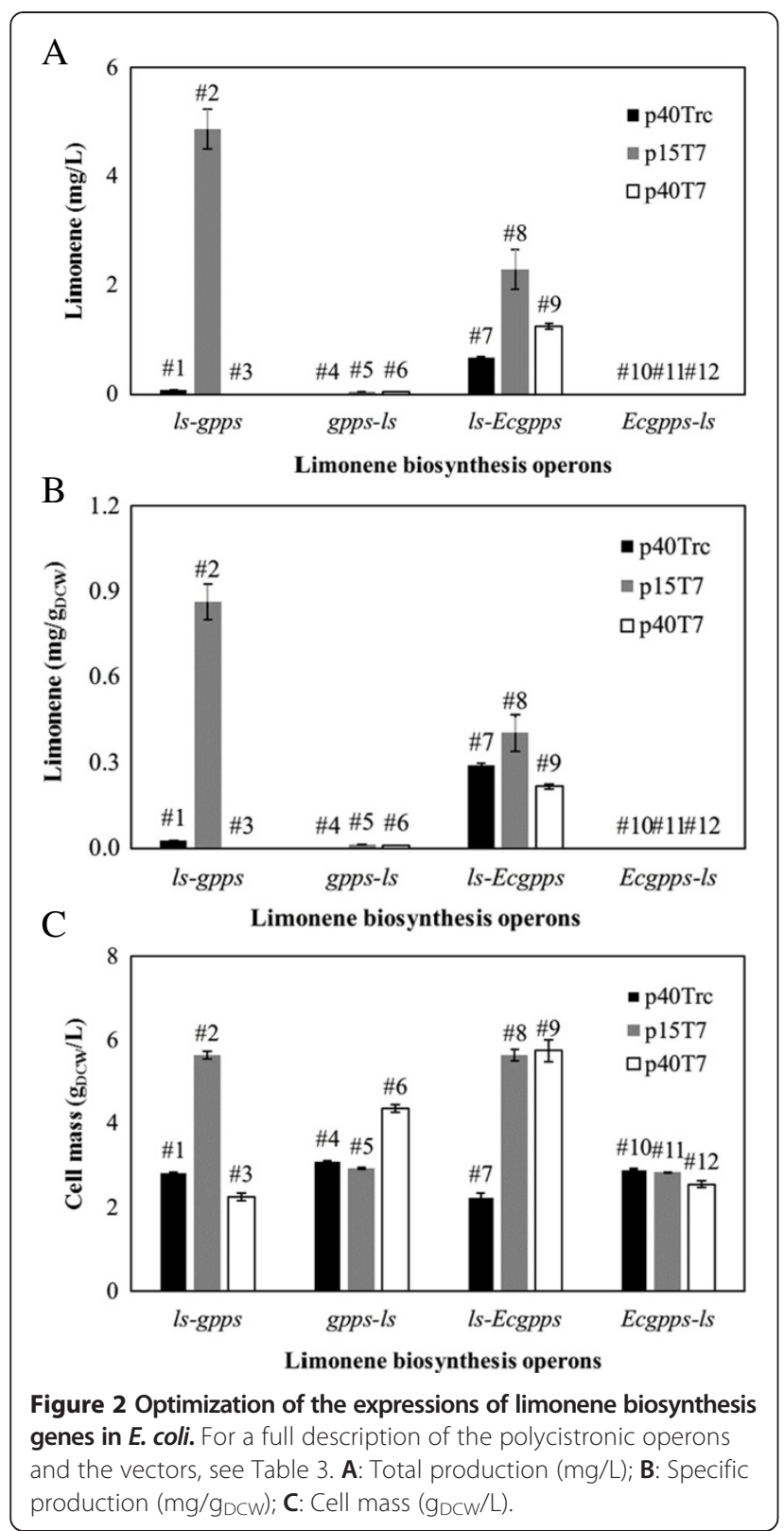

The reactions catalyzed by DXS and IDI have been reported as the most critical rate-limiting step in the endogenous MEP pathway of E. coli [27-30]. In this study, the polycistronic operon $d x s$-idi was constructed based on pTrcHis2B, pQE30 and pET21a + to form p40Trc-dxs-idi, p40T5- $d x s-i d i$ and $\mathrm{p} 40 \mathrm{~T} 7-d x s-i d i$. These three plasmids were respectively introduced into the strain $\# 2$, resulting in another three strains designated as strains \#13, \#14 and $\# 15$. Then the strains \#2, \#13, \#14 and \#15 were cultured in 2YT medium for $48 \mathrm{~h}$. Various inducer (IPTG) concentrations, from $10 \mu \mathrm{M}$ to $500 \mu \mathrm{M}$, were also evaluated. As shown in Figure 3, the strain \#13 containing both plasmids p40T7-dxs-idi and p15T7-ls-gpps could accumulate limonene up to $17.4 \mathrm{mg} / \mathrm{L}$ (or $2.89 \mathrm{mg} / \mathrm{g}_{\mathrm{DCW}}$ ) after $48 \mathrm{~h}$
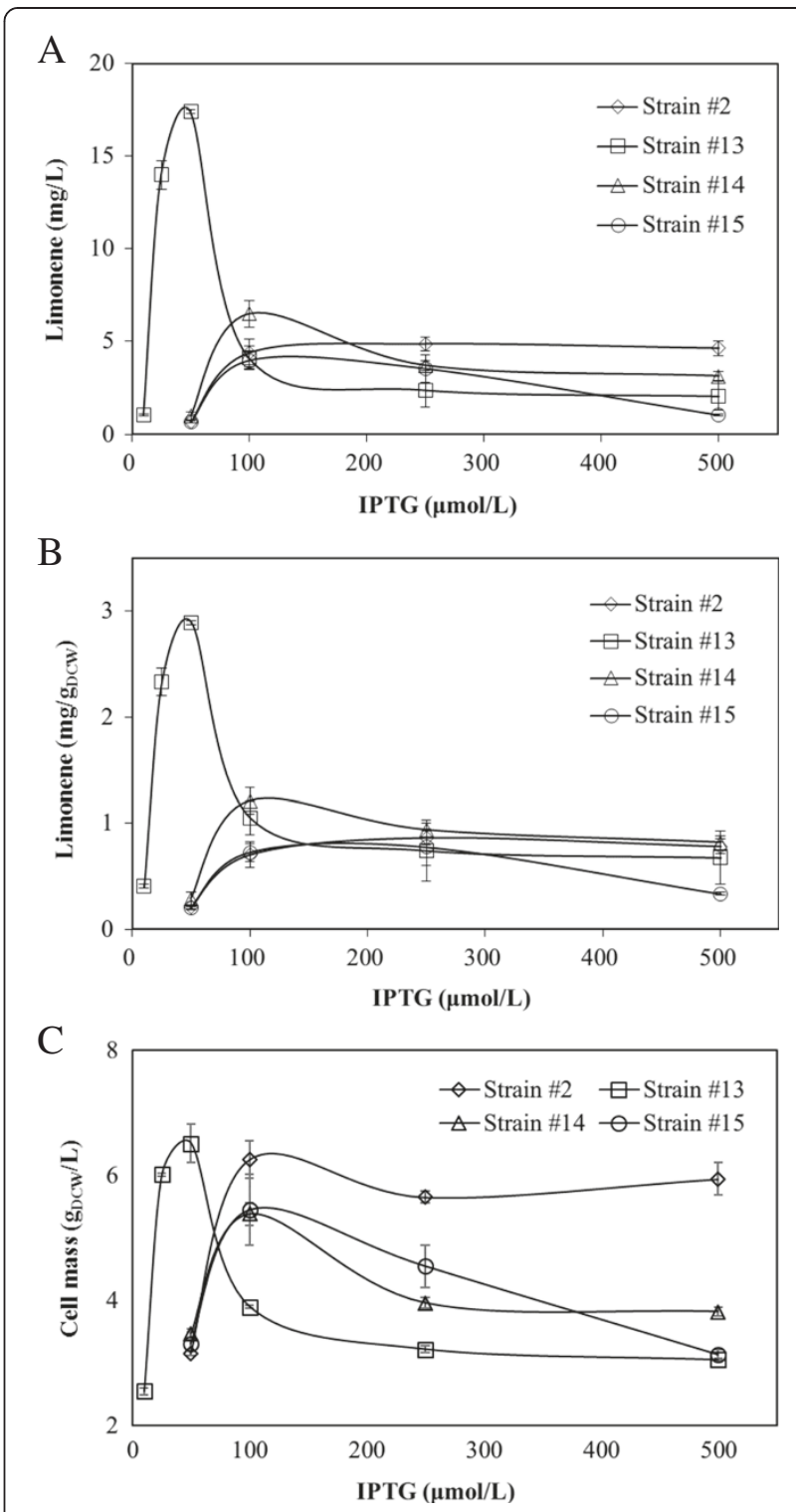

Figure 3 Overexpression of two rate-limiting enzymes in the endogenous MEP pathway to supply building blocks. For a full description of the strains and their plasmids, see Table 3 and Table 1, respectively. A: Total production ( $\mathrm{mg} / \mathrm{L})$. B: Specific production $\left(\mathrm{mg} / \mathrm{g}_{\mathrm{Dcw}}\right) ; \mathbf{C}$ : Cell mass (gDcw/L).

cultivation with IPTG induction at $50 \mu \mathrm{M}$, which represents approximately a 3.6 -fold enhancement in total limonene production (from 4.87 to $17.4 \mathrm{mg} / \mathrm{L}$ ) or a 3.3fold improvement in specific production (from 0.863 to $\left.2.89 \mathrm{mg} / \mathrm{g}_{\mathrm{DCW}}\right)$. Meanwhile the cell mass was the largest. Additionally, limonene production significantly affected the cell mass and glycerol consumption (Figure 3C, D). Clearly, the higher productivity and more robust growth of strain \#13 allowed higher limonene accumulation. Further improvements should be possible through medium optimization. 


\section{Effects of basis media and pyruvate on limonene production}

Besides the genetic modulation described above, we also optimized the growth medium to further increase the limonene productivity. E. coli strain \#13 was respectively cultured in 2YT, LB or TB media. The limonene production was measured after $24 \mathrm{~h}, 48 \mathrm{~h}$ and $72 \mathrm{~h}$ cultivation. Although the three media had no significant differences in the specific production of limonene, $2 \mathrm{YT}$ medium gave higher titers than the other two media (Figure 4). So 2YT medium was chosen as the basis medium for the cultivation of genetically engineered strain \#13. In a previous work [24], pyruvate and dipotassium phosphate were found beneficial for isoprenoid production in $E$. coli. Therefore, the 2YT medium supplemented with different concentrations of pyruvate was designed and employed for the culture of strain \#13. As shown in Figure 5, addition of $4 \mathrm{~g} / \mathrm{L}$ pyruvate could stimulate the limonene production of up to $15.1 \mathrm{mg} / \mathrm{L}$ or $4.11 \mathrm{mg} / \mathrm{g}_{\mathrm{DCW}}$ after 24 h cultivation, $32.5 \mathrm{mg} / \mathrm{L}$ or $5.59 \mathrm{mg} / \mathrm{g}_{\mathrm{DCW}}$ after $48 \mathrm{~h}$, and $35.8 \mathrm{mg} / \mathrm{L}$ or $5.76 \mathrm{mg} / \mathrm{g}_{\mathrm{DCW}}$ after $72 \mathrm{~h}$. Although the cell mass was basically the same (Figure $5, \mathrm{C}$ ), it is approximately a 2 -fold increase in limonene production as compared with the un-optimized medium used in this work or a 7-fold increase in contrast to the initial titer $(4.87 \mathrm{mg} / \mathrm{L})$. Meanwhile, the consumption of glycerol was also the largest (Figure 5, D). In addition, the effect of $\mathrm{K}_{2} \mathrm{HPO}_{4}$ was also examined (data not shown), although the improvement was not so significant as pyruvate.

\section{Discussion}

In this paper, we significantly increased the limonene yield in $E$. coli by optimizing the expression of the two limonene biosynthesis genes, overexpression of the two rate-limiting enzymes in the endogenous MEP pathway, and optimization of the culture medium (Figure 6). We established a limonene biosynthesis pathway in E. coli using four different polycistronic operons based on three vectors with varied expression strength. Previous researches on limonene biosynthesis focused merely on the expression of a certain polycistronic operon (e.g., gpps-ls) based on a specified vector $[3,4]$. In addition, only the GPPS from plant Abies grandis was tried for limonene production $[3,4]$. In this study, Ecgpps, a bacterial gpps gene that is native to $E$. coli, was tried for the first time for limonene production. Previous study indicated that limonene biosynthesis pathway flux was limited by the inherently low enzyme activity of the plant-originated GPPS [4]. However, our result showed that the protein-engineered $E c F S_{S 80 F}$ from $E$. coli also suffers a problem of low activity for limonene production. The two genes connected by IRES sequences can be expressed from a single promoter
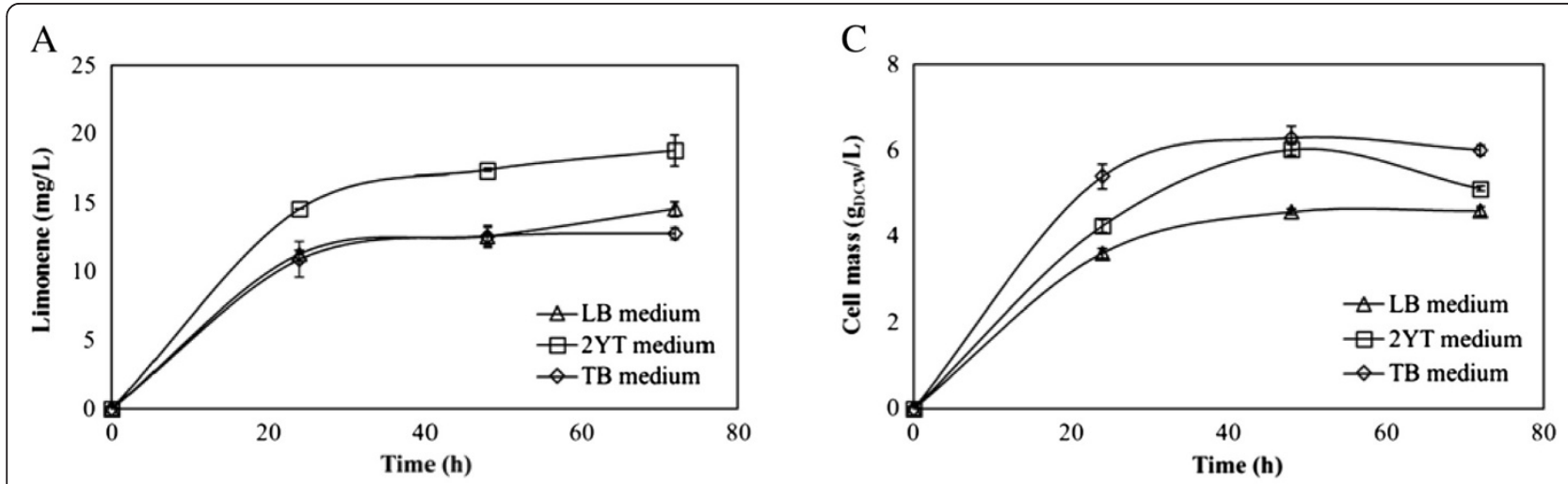

B
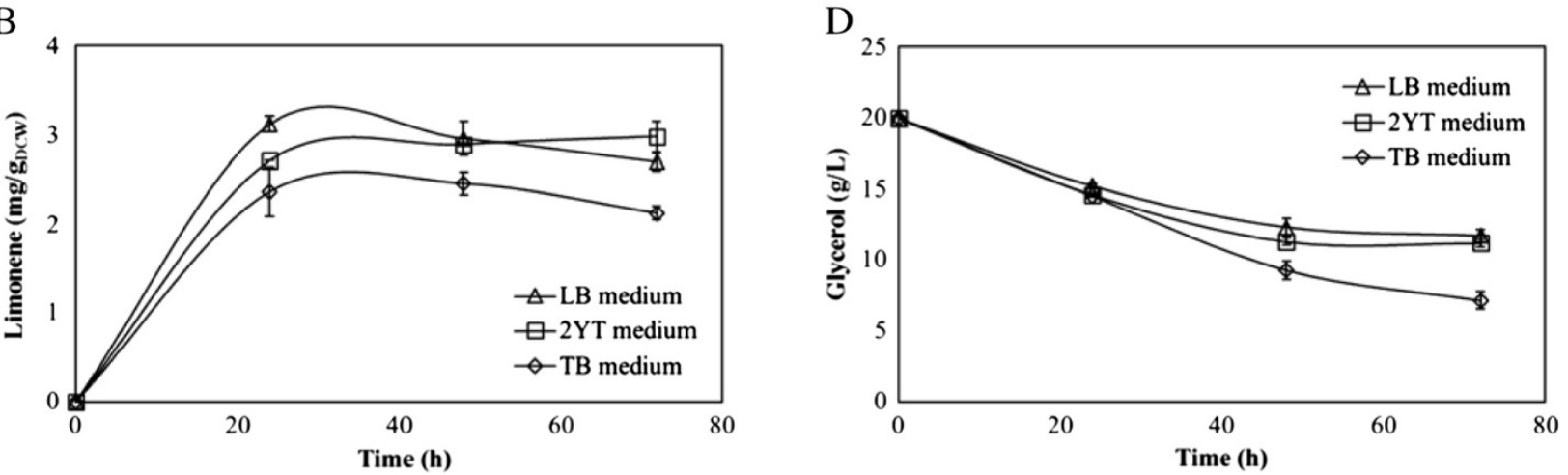

Figure 4 Effect of culture medium on limonene production of the strain \#13. A: Total production (mg/L); B: Specific production (mg/gDcw); C: Cell mass ( $\left.g_{D C w} / L\right)$; D: Glycerol in medium $(g / L)$. 

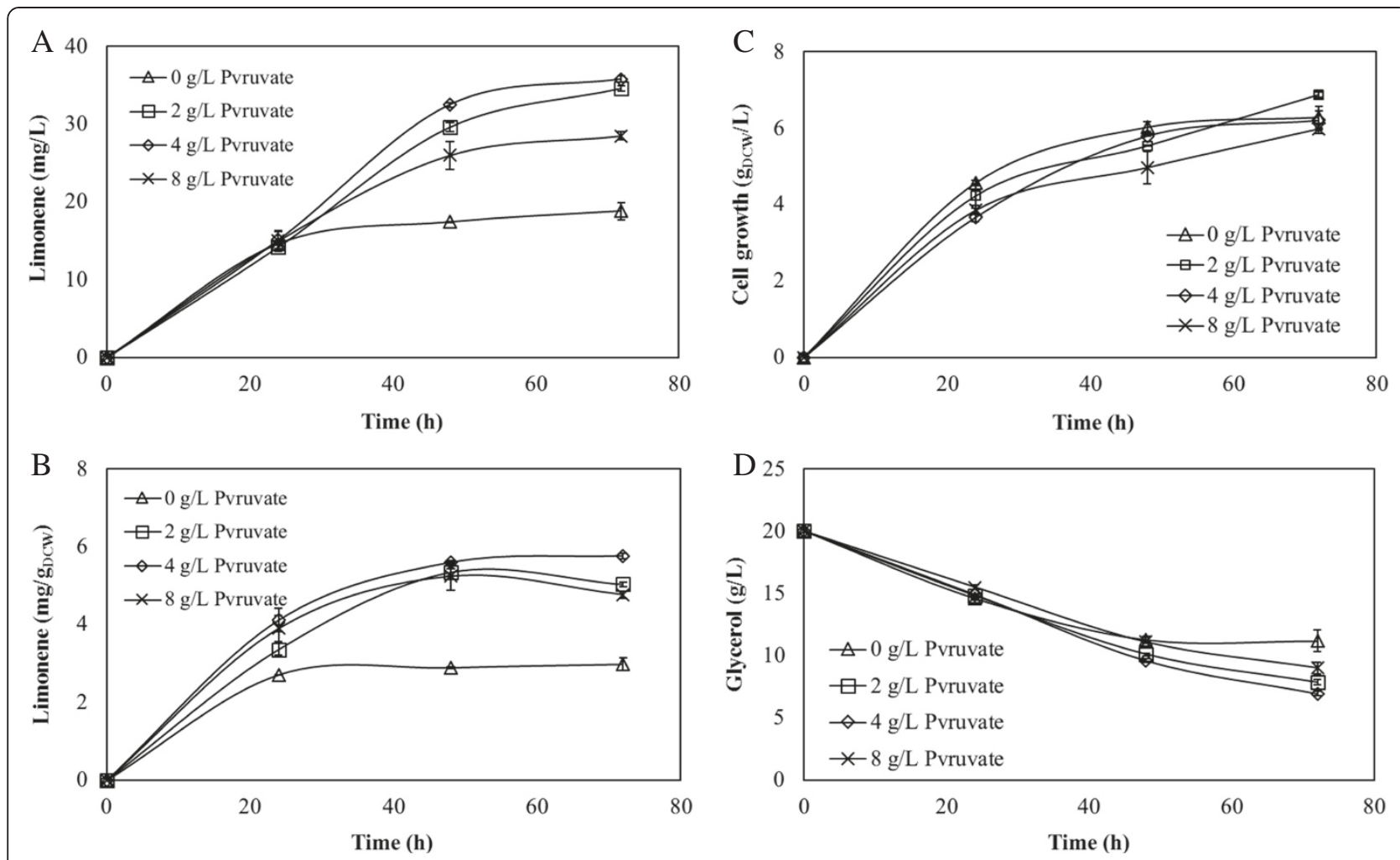

Figure 5 Effect of pyruvate concentration on limonene production of the strain \#13. A: Total production (mg/L); B: Specific production (mg/gDcw); C: Cell mass (gDcw/L); D: Glycerol in medium (g/L).

in IRES method used in this study. In the prokaryotic expression system, the gene ranked in front will be transcripted and translated primarily, followed by the transcription and translation of the subsequent gene in the polycistronic operons [31]. So the order of the limiting genes in polycistronic operons has a lot to do with the expression of the genes and the quantity of final product [14,15,32]. Our result showed that those E. coli strains expressing ls-gpps or ls-Ecgpps produced larger amounts of limonene than the strains expressing gpps-ls or Ecgpps-ls. This result disputes the current notion that the order of the limiting genes in polycistronic operons should correspond to that of terpenoid metabolic pathway in the natural and fine regulation mechanism of microorganisms [19]. In addition, the expression strength of vectors used for limonene biosynthesis was also indicated to be a very important determinant for the final limonene yield.

Our work represents the first report on limonene biosynthesis via overexpression of rate-limiting enzymes in MEP pathway. When the two limiting genes $(d x s \in \mathcal{E}$ idi) were coexpressed, both the expression strength and IPTG concentration were very important for achieving a high

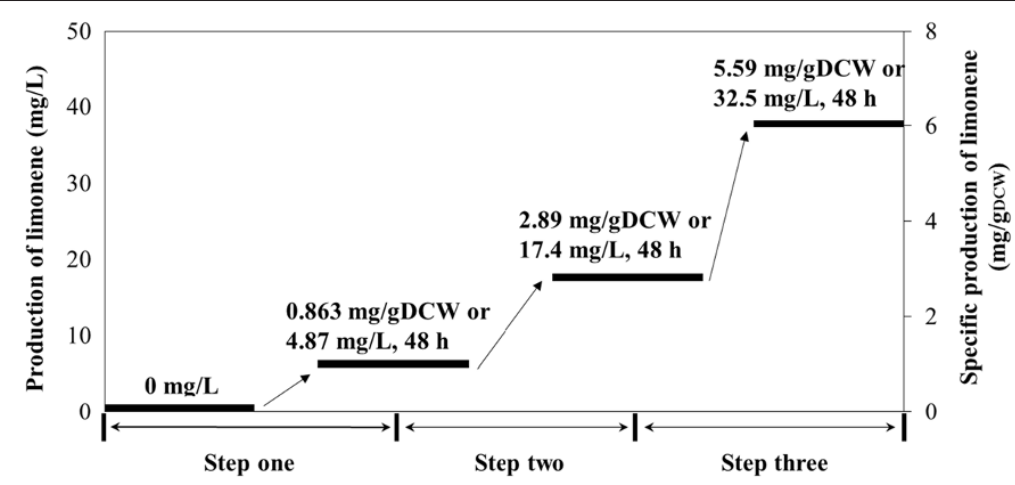

Figure 6 Progressive enhancement of limonene production in E. coli. The limonene yield of E. coli was increased by three steps: Optimization of the limonene biosynthesis genes expression; overexpression of rate-limiting enzymes in the endogenous MEP pathway; and medium adjustment. 
limonene yield. The polycistronic operon $d x s$-idi was expressed on various vectors, which resulted in different limonene productions. This phenomenon was also reported in the researches on taxadiene and levopimaradiene biosynthesis $[14,15]$. Once again, an appropriate expression strength of genes made tremendous contribution to the overall yield of the final product. Although a direct comparison of limonene yield with previous studies on isoprene or terpenoid is difficult because of the differences in products and assay conditions used, our results agree well with the published values of increases in isoprene or terpenoid yields when overexpressing enzymes of MEP pathway (Table 4). Although it might have become a popular point that the MEP pathway is not effective for high-level production of terpenoid $[9,33]$, the MEP pathway is a native pathway and the stoichiometry of MEP pathway is more efficient as compared to MVA pathway for IPP/DMAPP synthesis [34]. The limonene production by the MEP pathway in this work was much lower than the reported result by the MVA pathway [3]. It was partly because only two bottleneck enzymatic steps ( $d x s$ and $i d i)$ were targeted to increase the flux through the MEP pathway. Previous work [35] revealed that ispB, ispD and isp $F$ also produce rate-limiting enzymes impacting the MEP flux. Second, previous results showed that the expression of B. subtilis bottleneck enzymes in E. coli resulted in an enhancement of total isoprene production [17]. The low enzyme activity and the native regulation of the native bottleneck enzymes might be the reasons of low limonene production in this work. Third, different E. coli strains might result in the difference in limonene biosynthesis. E. coli BL21 (DE3) might not be as suitable as E. coli DH1 for terpene biosynthesis [3].

Besides genetic modulations, we also systematically optimized the culture medium to further increase limonene production. Pyruvate and dipotassium phosphate were chosen as supplements. The addition of pyruvate as the auxiliary carbon source has been reported to increase terpenoid production [24]. Since pyruvate was the immediate precursor of MEP pathway, the addition of pyruvate greatly increased the DMAPP synthesis so as to significantly enhance the limonene production. In addition, the addition of pyruvate affects the main metabolism. Namely, keto-acid such as pyruvate, OAA, and 2KG etc. affect Cya and in turn affect cAMP level. In the case of using glycerol as a carbon source, cAMP-Crp level is high in the wild type, while this may be decreased when pyruvate was added, and thus the glycerol consumption rate may be affected, since glpFKD is under control of cAMP-Crp. Dipotassium phosphate was also found to be beneficial for limonene production in E. coli. It supports the results of the previous study on terpenoid biosynthesis [24].

To prevent limonene volatilizing and to extract it from the culture medium, a two-phase culture system composed of $n$-hexadecane was adopted in this study. Hexadecane was chosen for its high hydrophobicity $\left(\log \mathrm{P}_{\mathrm{O} / \mathrm{W}}, 8.8\right)$ for the extraction of limonene, and low volatility, which prevents loss due to evaporation. A ratio of $1 / 50\left(V_{\mathrm{org}} / V_{\mathrm{aq}}\right.$, $2 \mathrm{~mL}$ of hexadecane was layered over $100 \mathrm{~mL}$ of culture

Table 4 The terpenoid production increased in this study and previous studies

\begin{tabular}{|c|c|c|c|}
\hline Products & Production increased & Genes overexpressed & References \\
\hline Isoprene & 0.4 -fold & $d x s$ and $d x r^{a}$ & Xue et al. [16] \\
\hline Phytoene & 2.1-fold & idi & Kajiwara et al. [36] \\
\hline Isoprene & 2.3-fold & $d x s$ and $d x r$ & Zhao et al. [17] \\
\hline$\beta$-Carotene & 2.7-fold & idi & Kajiwara et al. [36] \\
\hline Abietadiene & 2.8-fold & $d x s, d x r$ and idi & Morrone et al. [33] \\
\hline Squalene & 2.9-fold & $d x s$ and idi & Ghimire et al. [37] \\
\hline Limonene & 3.4-fold & $d x s$ and idi & This work \\
\hline Carotenoids & 3.5 -fold & $d x s, d x r$ and idi & Albrecht et al. [38] \\
\hline Isoprene & 4.0-fold & $d x s, i s p G^{b}, i s p H^{c}, i d i, i s p E^{d}, d x r, i s p D^{e}$ and isp $F^{f}$ & Zurbriggen et al. [18] \\
\hline Lycopene & 4.5 -fold & idi & Kajiwara et al. [36] \\
\hline Isoprene & 4.8-fold & $d x s, d x r$ and $i d i$ & Lv et al. [19] \\
\hline Taxadiene & 15.6-fold & $d x s, i d i$ ispD and ispF & Ajikumar et al. [14] \\
\hline Levopimaradiene & 611.3-fold & $d x s$, idi ispD and ispF & Leonard et al. [15] \\
\hline
\end{tabular}

The rate-limiting enzymes in the MEP pathway were overexpressed in all the studies listed in this table.

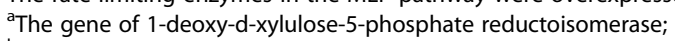

${ }^{\mathrm{b}}$ The gene of $(E)$-4-hydroxy-3-methyl-but-2-enyl pyrophosphate synthase;

${ }^{\mathrm{C}}$ The gene of (E)-4-hydroxy-3-methyl-but-2-enyl pyrophosphate reductase;

${ }^{\mathrm{d}}$ The gene of 4-diphosphocytidyl-2-c-methyl-d-erythritol kinase;

${ }^{\mathrm{e}}$ The gene of 2-c-methyl-d-erythrtol-4-phosphate cytidyltransferase;

${ }^{f}$ The gene of 2-c-methyl-d-erythritol 2, 4-cyclodiphosphate synthase. 
broth) was adopted in this two-phase culture system. Limonene was extracted into the hexadecane phase, and negligible amounts of limonene were detected in the cell mass and culture broth (data not shown). As a result, limonene production was measured only from the hexadecane phase. The two-phase culture system using hydrophobic and low volatile organic solvent was a very useful and common method used in terpenoid biosynthesis $[3,14,15]$.

\section{Conclusions}

Although the limonene production was enhanced significantly in this study, more efforts are still needed to achieve a higher production and to make it more economic. On one hand, the metabolic flux of the endogenous MEP pathway can be enhanced by overexpressing the rate-limiting enzymes from other bacteria such as Bacillus subtilis [17] and/or blocking the competing metabolic pathways. On the other hand, the problem of low GPPS/LS activities needs also to be addressed by directed evolution or rational protein engineering. Besides, the culture medium should be more efficient and economic, and the metabolically engineered strain should be cultivated in a bioreactor for commercial production of limonene in the future.

\section{Competing interests}

The authors declare that they have no competing interests.

\begin{abstract}
Authors' contributions
F-LD has made substantial contributions to conception and design, and acquisition of data, and analysis and interpretation of data; H-LY has been involved in drafting the manuscript and revising it critically for important intellectual content; J-HX has given final approval of the version to be published; C-XL has made substantial contributions to design and has been involved in drafting the manuscript and revising it critically for important intellectual content. All authors read and approved the final manuscript.
\end{abstract}

\section{Acknowledgements}

This work was financially supported by the National Natural Science Foundation of China (No. 21276082), Ministry of Science and Technology, P. R. China (Nos. 2011CB710800), and Shanghai Commission of Science and Technology (No. 11431921600). The authors are grateful to Dr. Yunpeng Bai for proof-reading of the manuscript and to professor Hongwei Yu at

Zhejiang University for his generous gift of vectors and constructive advices.

Received: 8 May 2014 Revised: 12 July 2014 Accepted: 17 July 2014 Published online: 31 August 2014

\section{References}

1. Misawa N (2011) Pathway engineering for functional isoprenoids. Curr Opin Biotechnol 22:627-633

2. Colby SM, Alonso WR, Katahira EJ, Mcgarvey DJ, Croteau R (1993) 4S-Limonene synthase from the oil glands of spearmint (Mentha spicata) CDNA isolation, characterization, and bacterial expression of the catalytically active monoterpene cyclase. J Biol Chem 268:23016-23024

3. Alonso-Gutierrez J, Chan R, Batth TS, Adams PD, Keasling JD, Petzold CJ, Lee TS (2013) Metabolic engineering of Escherichia coli for limonene and perillyl alcohol production. Metab Eng 19:33-41

4. Carter OA, Peters RJ, Croteau R (2003) Monoterpene biosynthesis pathway construction in Escherichia coli. Phytochemistry 64:425-433

5. Duetz WA, Bouwmeester H, Beilen JB, Witholt B (2003) Biotransformation of limonene by bacteria, fungi, yeasts, and plants. Appl Microbiol Biotechnol 61:269-277
6. Keasling JD (2010) Manufacturing molecules through metabolic engineering. Science 330:1355-1358

7. Tracy NI, ChenD CDW, Price GL (2009) Hydrogenated monoterpenes as diesel fuel additives. Fuel 88:2238-2240

8. Campos N, Rodriguez-Concepcion M, Sauret-Gueto S, Gallego F, Lois LM, Boronat A (2001) Escherichia coli engineered to synthesize isopentenyl diphosphate and dimethylallyl diphosphate from mevalonate: a novel system for the genetic analysis of the 2-c-methyl-d-erythritol-4-phosphate pathway for isoprenoid biosynthesis. Biochem J 353:59-67

9. Martin VJ, Pitera DJ, Withers ST, Newman JD, Keasling JD (2003) Engineering a mevalonate pathway in Escherichia coli for production of terpenoids. Nat Biotechnol 21:796-802

10. Pitera DJ, Paddon CJ, Newman JD, Keasling JD (2007) Balancing a heterologous mevalonate pathway for improved isoprenoid production in Escherichia coli. Metab Eng 9(2):193-207

11. Hunter WN (2007) The non-mevalonate pathway of isoprenoid precursor biosynthesis. J Biol Chem 282(30):21573-21577

12. Croteau RB, Davis EM, Ringer KL, Wildung MR (2005) (-)-Menthol biosynthesis and molecular genetics. Naturwissenschaften 92:562-577

13. Burke C, Croteau R (2002) Geranyl diphosphate synthase from Abies grandis: CDNA isolation, functional expression, and characterization. Arch Biochem Biophys 405:130-136

14. Ajikumar PK, Xiao WH, Tyo KEJ, Wang Y, Simeon F, Leonard E, Mucha O, Phon TH, Pfeifer B, Stephanopoulos G (2010) Isoprenoid pathway optimization for taxol precursor overproduction in Escherichia coli. Science 330:70-74

15. Leonarda E, Ajikumara PK, Thayer K, Xiao WH, Mo JD, Tidorb B, Stephanopoulosa G, Thayer K, Prather KL (2010) Combining metabolic and protein engineering of a terpenoid biosynthetic pathway for overproduction and selectivity control. Proc Natl Acad Sci 107:13654-13659

16. Xue J, Ahring BK (2011) Enhancing isoprene production by genetic modification of the 1-deoxy-d-xylulose-5-phosphate pathway in Bacillus subtilis. Appl Environ Microbiol 77:2399-2405

17. Zhao Y, Yang J, Qin B, Li Y, Sun Y, Su S, Xian M (2011) Biosynthesis of isoprene in Escherichia coli via methylerythritol phosphate (MEP) pathway. Appl Microbiol Biotechnol 90:1915-1922

18. Zurbriggen A, Kirst H, Melis A (2012) Isoprene production via the mevalonic acid pathway in Escherichia coli (bacteria). BioEnergy Research 5:814-828

19. Lv X, Xu H, Yu H (2013) Significantly enhanced production of isoprene by ordered coexpression of genes $d x s, d x r$, and idi in Escherichia coli. Appl Microbiol Biotechnol 97:2357-2365

20. Williams DC, McGarvey DJ, Katahira EJ, Croteau R (1998) Truncation of limonene synthase preprotein provides a fully active 'pseudomature' form of this monoterpene cyclase and reveals the function of the amino-terminal arginine pair. Biochemistry 37:12213-12220

21. Brosius J, Erfle M, Storella J (1985) Spacing of the -10 and -35 regions in the tac promoter: Effect on its in vivo activity. J Biol Chem 260:3539-3541

22. Brunner M, Bujard $H$ (1987) Promoter recognition and promoter strength in the Escherichia coli system. EMBO J 6:3139-3144

23. Jang HJ, Yoon SH, Ryu HK, Kim JH, Wang CL, Kim JY, Oh DK, Kim SW (2011) Retinoid production using metabolically engineered Escherichia coli with a two-phase culture system. Microb Cell Fact 10:59

24. Zhou K, Zou R, Zhang C, Stephanopoulos G, Too HP (2013) Optimization of amorphadiene synthesis in Bacillus subtilis via transcriptional, translational, and media modulation. Biotechnol Bioeng 110:2556-2561

25. Song HB, Demain AL (1977) An improved colorimetric assay for polyols. Anal Biochem 81(1):18-20

26. Reiling KK, Yoshikuni Y, Martin VJ, Newman J, Bohlmann J, Keasling JD (2004) Mono and diterpene production in Escherichia coli. Biotechnol Bioeng 87:200-212

27. Lois LM, Rodriguez-Concepcion M, Gallego F, Campos N, Boronat A (2000) Carotenoid biosynthesis during tomato fruit development: regulatory role of 1-deoxy-d-xylulose-5-phosphate synthase. Plant J 22(6):503-513

28. Miller B, Heuser T, Zimmer W (2000) Functional involvement of a deoxyxylulose-5-phosphate reductoisomerase gene harboring locus of Synechococcus leopoliensisin isoprenoid biosynthesis. FEBS Lett 481:221-226

29. Estevez JM, Cantero A, Reindl A, Reichler S, Leon P (2001) 1-Deoxy$\mathrm{d}$-xylulose-5-phosphate synthase, a limiting enzyme for plastidic isoprenoid biosynthesis in plants. J Biol Chem 276(25):22901-22909

30. Das A, Yoon SH, Lee SH, Kim JY, Oh DK, Kim SW (2007) An update on microbial carotenoid production: application of recent metabolic engineering tools. Appl Microbiol Biotechnol 77(3):505-512 
31. Zaslaver A, Mayo AE, Rosenberg R, Bashkin P, Sberro H, Tsalyuk M, Surette MG, Alon U (2004) Just-in-time transcription program in metabolic pathways. Nat Genet 36:486-491

32. Nishizaki T, Tsuge K, Itaya M, Doi N, Yanagawa H (2007) Metabolic engineering of carotenoid biosynthesis in Escherichia coli by ordered gene assembly in Bacillus subtilis. Appl Environ Microbiol 73(4):1355-1361

33. Morrone D, Lowry L, Determan MK, Hershey DM, Xu M, Peters RJ (2010) Increasing diterpene yield with a modular metabolic engineering system in E. coli: comparison of MEV and MEP isoprenoid precursor pathway engineering. Appl Microbiol Biotechnol 85(6):1893-1906

34. Meng H, Wang Y, Hua Q, Zhang S, Wang X (2011) In silico analysis and experimental improvement of taxadiene heterologous biosynthesis in Escherichia coli. Biotechnol Bioprocess Eng 16(2):205-215

35. Yuan LZ, Rouvière PE, Larossa RA, Suh W (2006) Chromosomal promoter replacement of the isoprenoid pathway for enhancing carotenoid production in E. coli. Metab Eng 8(1):79-90

36. Kajiwara S, Fraser PD, Kondo K, Misawa N (1997) Expression of an exogenous isopentenyl diphosphate isomerase gene enhances isoprenoid biosynthesis in Escherichia coli. Biochem J 324:421-426

37. Ghimire GP, Lee HC, Sohng JK (2009) Improved Squalene Production via Modulation of the Methylerythritol 4-Phosphate Pathway and Heterologous Expression of Genes from Streptomyces peucetius ATCC 27952 Escherichia coli. Appl Environ Microbiol 75(22):7291-7293

38. Albrecht M, Misawa N, Sandmann G (1999) Metabolic engineering of the terpenoid biosynthetic pathway of Escherichia coli for production of the carotenoids $\beta$-carotene and zeaxanthin. Biotechnol Lett 21(9):791-795

doi:10.1186/s40643-014-0010-z

Cite this article as: Du et al.: Enhanced limonene production by optimizing the expression of limonene biosynthesis and MEP pathway genes in E. coli. Bioresources and Bioprocessing 2014 1:10.

\section{Submit your manuscript to a SpringerOpen ${ }^{\circ}$ journal and benefit from:}

- Convenient online submission

- Rigorous peer review

- Immediate publication on acceptance

- Open access: articles freely available online

- High visibility within the field

- Retaining the copyright to your article

Submit your next manuscript at $\gg$ springeropen.com 